\title{
FIRST RESPONDER INFORMATION FLOW SIMULATION: A TOOL FOR TECHNOLOGY ASSESSMENT
}

\author{
C. Donald Robinson \\ Donald E. Brown \\ University of Virginia \\ Department of Systems and Information Engineering \\ P.O. Box 400747 \\ 151 Engineer's Way \\ Charlottesville, VA 22904
}

\begin{abstract}
New information technology is becoming available for first responders at a rapid rate. Often this new technology comes with not only new capabilities but also a high price tag. Policy makers and emergency managers need a way to assess which portfolio of technologies achieves the greatest benefit at a minimal cost. Assessing these technologies in a real world setting not only takes a long time but is also often cost-prohibitive. Thus, using computer simulation to assess the benefits of new technologies would yield significant insight into the usefulness of new technologies in a cost and time-efficient manner. A computer simulation combining an agent-based architecture and GIS information called Disaster Response Information Flow and Technology Simulator (DRIFTS) is developed to model the information flow in a disaster setting. DRIFTS, combined with a response surface methodology, can be used to assess different types of information technologies for disaster response.
\end{abstract}

\section{INTRODUCTION}

With the ever increasing interest in bettering our nation's preparedness for disasters comes a seemingly innumerable selection of available technologies to aid in that preparedness and subsequent emergency response. Many of these new technologies give similar benefits at often different costs. Therein lies the necessity of properly assessing not only the capabilities of these technologies but also the relative benefit that they provide not only to the responders but also to the potential victims of disaster. Given the unfortunate finite budgets that emergency managers must operate with and the tremendous responsibility placed on the shoulders of the responders and their technology, there must be objective and thorough analysis of which portfolio of technologies best serves potential victims of disaster.

Two of the greatest problems associated with assessing technology for emergency response are the inherent rarity of the events which necessitate the use of this technology, and the prohibitive cost associated with real-world testing. Live simulations of disasters often cost tens of thousands of dollars and in such a live simulation one might only be able to test a handful of new technologies. Thus there exists a need for computer simulation in technology assessment.

There is an even further need for testing new information technology for use in emergency response. As our world progresses through the information age, our agencies must be able to cope with the ostensibly infinite amount of data available. These agencies must not only be able to receive the appropriate data, but also to turn it into correct information and then transform that information into knowledge. This information and knowledge must then be conveyed quickly and efficiently to potential users of that knowledge or information.

Given the necessity of computer simulation and the need to assess the efficacy of new information technologies, the actual information flow in a disaster scenario must be modeled and simulated appropriately. This information flow is the collection of data in the response environment, the fusing of that data into information, and the dissemination of that information to other agents within the simulated environment. With a robust and flexible environment which models this information flow effectively, new and existing information technologies can be assessed for their costs and benefits to society.

\section{BACKGROUND}

Many have explored various areas of research in disaster simulation. Jain, McLean and Leong (2003) provide an in depth survey of available simulation materials in this area. In fact, many have recognized the place for modeling and simulation in emergency response for many years. Sullivan (1985) provides a report on the subject. Wallace and Balogh (1985) also provide an example of using information 


\section{Robinson and Brown}

technology in disaster response almost two decades ago. However, the idea of assessing that technology through computer simulation is a topic which requires much more research and testing.

Furthermore, there must also be a way of objectively interpreting the output of an emergency response simulator. The performance of the overall emergency response system must be assessed through some set of consistent metrics. These metrics must be able to demonstrate the overall change in output (effects of disaster response) given certain changes in the inputs (new technologies).

Measuring the performance of an entity like an emergency responder is a difficult task. There is no standardized method for obtaining data and information from an actual disaster (Guha-Sapir 2000). Furthermore, relying on data sets of disasters collected from various agencies leads to ambiguous terms, inconsistent and incomplete data, and general confusion (Guha-Sapir 2000). Thus, using real disaster data to assess an emergency response or to assess the effectiveness of certain technological inputs is tedious and incomplete at best.

Modeling and simulation provides an inexpensive and time-effective method of observing a system and also provides a way to test multiple inputs and evaluate different outputs. As Sanchez and Lucas (2002) suggest, one of the best reasons for implementing a simulation to observe a system is to, "leverage simulation's advantages in cost and time relative to many real-world experiments".

One challenge of integrating micro-scale simulations together to observe a system level progression of a disaster is the inherent disorder present in a disaster scenario. In an emergency response to a disaster the responders must undergo a change in overall strategy from typical day to day response, to disaster response. Disaster response involves more interagency collaboration as well as different tactics and methods of carrying out the overall mission of assistance to the affected population (Koehler 1995). This change can cause great deals of confusion and must be dealt with in the overall model of a simulation as well as the measurement of an emergency response. Modeling this confusion accurately requires validation of the model.

Validation and assessment of a simulation is critical (Balci 1998) (Knepell 1993). Validating a simulation of a chaotic event as a disaster is difficult due to the scarcity and inconsistencies in actual data for comparison. Similarly, assessing the validity of selected measurements of a disaster will require verification from various sources including not only what little data is present, but also expert review.

If a valid computer simulation of a disaster event is created, its outputs must be assessed. As Jain and McLean state regarding the challenges of a disaster simulation, "Interpretation of the simulation output data might not be a straightforward process. . . . it may not be clear what action should be taken based on the results" (Jain and McLean 2003). This seems to be a common problem in simulation of disasters and other events. Considerable effort has been expended in creating simulations. However, often the output is not clearly addressed. As stated in Jain and McLean (2003), the objective of response to a disaster is to "minimize the impact of disaster events on entities of interest". The specific metrics which describe the "impact" are not identified and are often just assumed. For true objective analysis of any simulation or actual response, a clear and defined method should be consistently used.

\section{INFORMATION TECHNOLOGY IN DISASTERS}

One of the basic assumptions of this paper is that there is a lot of information which needs to be properly interpreted and disseminated in a disaster. The interpretation of this information begins with first fusing relevant data to create such information. Furthermore, this data must be collected by various sensors in the response environment. These sensors might include seismic sensors, air quality sensors, or just human sensors reporting on observed casualties. Lastly this information must then be distributed to possible users of it such as rescue squads who need to pick up and transport casualties or HAZMAT teams who need to respond to a chemical spill.

\subsection{A Possible Scenario}

Consider a large wildfire where hundreds if not thousands of responders are present. Each fire fighter has an individual global positioning system (GPS) transmitter. Also, several of those fire fighters are positioned in the path of the fire and are not aware of it. These thousands of position transmissions must be first sent in a receivable and clear method. The data must then be processed into actual locations by some type of receiver. These locations must then be sent to a visualization unit combined with some type of mapping system such as one which uses a geographic information system (GIS) format. The location of the fire must also be detected through some type of technology such as an aerial infrared camera. A simulation-based decision support system (DSS) might then predict the current path of the fire given certain weather inputs and past locations of the fire. This simulation based DSS could be a combination of deterministic modeling such as some weather prediction programs as well as discrete-event or agent-based simulations which combine the weather, fire and containment elements of fire movement. The DSS might then alert a user that a number of fire fighters are in imminent danger. This user must then communicate through radio or some other wireless means to those firefighters in the path of the fire and they must then evacuate. This is a normative scenario of information technology being used in disaster response and one that does not seem too far outside of the realm of possibility. However, with 


\section{Robinson and Brown}

the countless number of choices for each element of technology in this scenario, which ones work best?

\subsection{Available Information Technologies}

The data streams available to emergency managers in historical disasters have been substantially smaller than what is currently available. Furthermore, it seems as though there is a seemingly exponential growth in the amount of data which must be processed.

The internet is a potential source for massive amounts of data and information relevant to a particular disaster. Emergency responders now have the ability to go to a web address and look up the particular seismic activity in some regions of the United States and see it almost real-time. Furthermore, more and more response agencies are obtaining the ability to access this enormous data source actually in the field (Jenvald 2001). Mobile satellite internet access terminals are continuing to be set up in command vehicles which will be deployed in any emergency. The terminals cost money and the satellite feed costs money and there is no current way to assess whether this money really benefits the potential victims of a disaster. This is not to say that providing internet access terminals to emergency response commanders does not help potential victims. In fact this may be one of the most effective information technologies available to responders. However, we have no way of determining the benefit.

Other potential sources of information for use in a disaster environment might be GPS transmitters placed on vehicles and/or personnel. With these, commanders could know at any given time where resources are being allocated and who might be in the greatest danger. While this technology might help in the case of the wildfire mentioned earlier, it might not be as useful where the responders are in buildings such as a large urban fire. Thus, particular technologies must be assessed with respect to the environment where they will be used.

There is also a problem with communications bandwidth for transmitting and reporting data. If every person responding to a disaster did have a small GPS transmitter, how would the positions be communicated to a command center? There are already bandwidth limitations for verbal wireless communication. Adding thousands of new transmissions to these frequencies would give commanders the desired positions of resources, but might limit the number of verbal communications. Is this desirable? Is it more important to receive data or verbal communication? These types of questions are the types that might be assessed with computer simulation.

\subsection{Data into Information}

Now consider that the data has been collected and transmitted to the appropriate users. This data must then be turned into information. An example of this can be shown using the weather data and wildfire scenario previously mentioned. In the scenario weather data was combined with previous fire locations to predict its future path. If a user receives data that the barometric pressure at a particular location is a certain level. This may not help a user very much. However, if this data was combined with previous data on barometric pressure and a trend emerges that the barometric pressure is falling, then this data can then be turned into information that there is a higher probability of rain which reduces the risk to the fire fighters in the path of the fire.

There are many ways to turn data into information. One of the most common methods is through statistical inference. Trends, patterns, and distributions can be derived from data points. These inferences lead to usable information for a responder. The example of a trend of decreasing barometric pressure as mentioned before shows how simple numbers can be turned into information about current and future weather conditions.

Data must also be turned into information efficiently. While many statistical techniques exist for obtaining information from data points, some work better than others. Consider a classroom of 10 five year old elementary school students with two 50 year old teachers in the room. A descriptive statistic of the age of the people in the room might be the average of all their ages. However, this would yield a value of 12.5. This is not necessarily very descriptive of the people in the room. The median, however, is 5 and this better describes the people in the classroom. Therefore, the method used to turn the data into information is very important for any type of information technology.

\section{THE USE OF SIMULATION}

As mentioned before, computer simulation has been recognized as important for emergency response for many years. Computer simulation can yield very cost effective and time efficient insight into emergency response operations. However, it cannot be used as a sole method of assessment and testing. While considerable effort has gone into models of human performance and response operations, these models are still incomplete. As George Box once said, "All models are wrong, but some are useful" (1979).

\subsection{Refining The Response Surface}

The outputs of an emergency response must first be clearly defined before any type of assessment can be done. However, for now, consider that there is one output and two inputs. While this is obviously simplistic for an emergency response, it helps conceptually in visualizing a response surface. This response surface is a simple plane which is assumed to have some bumps and valleys that represent optimal or non-optimal solutions. 


\section{Robinson and Brown}

Simulation alone cannot necessarily find the optimal solution. Because the models that are created always have some error, often substantial, from reality, the optimal solution that is found might not represent the best solution in practice. However, as mentioned before, testing in the real world gives a data point on that surface with less error, but at a much greater cost and fewer points can be assessed.

There is a hybrid solution of human-in-the-loop (HITL) simulation. This combines some models with actual human intervention providing the most accurate model of human performance and decision making. This solution seems to capture the best of computer simulation with reduced costs and modeling human performance. However, there are drawbacks to this method as well. While it is cheaper and faster than live simulations, it is still more expensive and slower than pure computerized simulation. It also has potentially lower error than pure computer simulation, but then again, the error is seemingly greater than that of live simulations.

While no one method of simulation seems to be far better than any other given multiple objectives, a combination of all three might be the best solution. Response surface methodology is a way to find an optimal point in a high dimensional surface with relatively few number of probed points on that surface. The basic concept is to probe points in a space filling design and fit a surface to the values obtained at those points. Then, given that fitted surface, identify a region of optimality and probe within that region to better fill its space. This procedure continues until a sufficient level of confidence is reached that the fitted optimum is in fact the optimal point on the actual surface (Myers and Montgomery 2002).

A possible way to assess information technology is to use a mixture of computer, HITL, and live simulation. Computer simulation would be used first to probe a large portion of the response environment. While computer simulation has a higher error from actual reality than the other two methods, it can inexpensively probe a larger portion of input options in a relatively short amount of time. From this sub-regions of possible optimality can then be found. In those sub-regions, HITL simulation would then be used. HITL would then find even smaller sub-regions of optimality. In this last and smallest sub-region, live simulation can be used to probe a few points to find the lowest variance optimal solution within the perceived optimal region. While it might initially seem excessive to try and implement all of these stages of simulation, in the end the solutions should be much better and there should be less waste in spending by using the advantages of all of these useful methods.

\subsection{Computer Simulation}

The first element of this methodology would involve computer simulation. With computer simulation lies another question of how the build the simulation. There are many accepted practices for simulation including discrete-event or agent-based design. There are other issues of interoperability and platforms to consider as well. Nevertheless, regardless of the method of computer simulation used, the simulation must accomplish the end goal of probing a large spectrum of inputs, assessing the values of the outputs, and doing this quickly and cheaply. Brady (2003) provides such an example of a discrete-event simulation which is able to quickly probe rare events in a rural part of Indiana where live simulation is costly and only examines a small portion of the possible threats that are present in that part of Indiana.

One of the aspects of computer simulation that is very desirable for testing is its ability to incorporate details of varying levels of fidelity. For instance, a rough discreteevent simulation can be created relatively quickly to test certain general ideas. These models can then be refined to include more detailed events which could represent reality a little better. Lastly computer models can be defined down to the individual agent level where individual human decisions are modeled and simulated.

This last type of simulation, agent-based, seems to be one of the most promising for modeling emergency response to a disaster. In an emergency response, the outcome is often the product of many individual decisions being made. Many of those decisions can have a significant effect on the outcome of subsequent events and then the overall response. Agent-based models handle this type of environment very well where the outcome of the simulation depends on individual decisions rather than aggregated processes.

Agent-based simulations also have the ability to be refined relatively quickly. Rough models of agent behavior can be implemented initially to begin the process. Then future validation and more modeling can be done to refine their behavior which in turn refines the simulation and increases its fidelity without significant change to the architecture or structure of the simulation. Gadomski et al. (1998) give a good method of using both a top-down and bottom-up approach to using computer simulation for modeling intelligent decision support-systems. This paper also gives evidence of the advantages of an agent-based system being used for refinement of more validated models.

Thus, in the proposed methodology of using computer, HITL and then live simulation, the use of computer simulation can be extended to first include coarse simulations and then later more refined simulations.

\section{D.R.I.F.T.S.}

Given the existing problem of a need to assess information technology within a disaster response scenario, an agentbased computer simulation called the Disaster Response Information Flow and Technology Simulation (DRIFTS) is under development. The simulation is designed to be very flexible and modular. It currently has many capabilities yet 


\section{Robinson and Brown}

as new models of performance become available, they can and will be easily integrated into the existing architecture.

\subsection{GIS Architecture}

The DRIFTS project is designed as an agent-based computer simulation with a GIS architecture. The GIS allows the simulation to correctly associate all geographic information in its environment. The system used in DRIFTS is ArcView and ESRI's MapObjects as an add in for Microsoft's Visual Basic. The capabilities of this architecture extend beyond that of just geographic association. This GIS system is vector based. It thus allows many different shapes, lines and points to be defined for different aspects of the simulation. For instance, a police car (point) has a viewing distance specified in the initialization of the program. As the police car moves along the roads (lines), it creates a buffer zone (polygon) of area that the policeman can see. Any casualty or damage within this buffer zone is then eligible to be reported. The agent-based architecture and GIS allow such a fine level of fidelity to be realized efficiently.

\subsection{Agent Layers}

The simulation is also designed to allow multiple agents to interact within the GIS framework. Each set of agents belongs to its own individual layer. For example, in the DRIFTS, the different agent layers include police, ambulances, roads, emergency support facilities, hospitals, and casualties. Each layer has its own behavior and set of possible actions. Additionally, any one layer can interact with any other layer in many ways. In the DRIFTS simulation the ambulances transport agents in the casualty layer and then drop them off with an agent in the hospital layer. The police report on damage in the road layer and in the emergency support facility and hospital layers. Furthermore, these reports help direct the ambulances on their way to pick up more casualties. While these are just a few of the interactions in the DRIFTS, many more are currently used and even more are possible.

Another benefit of the layering method is the efficiency of interactions. For instance, if an agent in the hospital layer never interacts with an agent in the road layer, then there is no need to for one to receive and process the information from the other. Thus, an agent only retrieves the necessary information from another layer thus reducing a significant memory burden from having to make sure every set of agents has total knowledge of the simulation environment.

\subsection{Using HAZUS for Loss Estimation}

The primary disaster that is currently used in the DRIFTS is an earthquake. More specifically it is modeled very closely to the 1994 earthquake that occurred in Northridge,
CA. The DRIFTS does not actually model the losses of both people and property due to this earthquake. For this the Federal Emergency Management Agency's HAZUS program is used. HAZUS produces a loss estimate per census tract in a given region. A user inputs into HAZUS data like where the epicenter should be located, the magnitude of the earthquake, the infrastructure that exists, etc. and HAZUS produces these loss estimates. A loss estimate is simply a proportion of people or property that sill sustain varying levels of damage.

What DRIFTS does is take these proportions and combines them with actual census data for each census tract. This census data gives the total number of people in each tract of a given demographic at a given time. This data is then combined with the various proportions of potential casualties to give a true number of the actual casualties within a region. These are then given specific locations within their own layer. Furthermore, the casualties are laid down with respect to other features in the data, such as roadways to create a realistic "ground truth" of the effects of the disaster. This "ground truth" then becomes the genesis of the simulation.

\subsection{Information Flow}

One of the main purposes of DRIFTS is clearly to model the information flow in a disaster scenario. Disasters always leave some degree of chaos in their wake which disrupts typical day to day information flow. Furthermore, disaster response often necessitates more information about a particular environment than is normal. Thus, these two aspects must be taken into account.

One of the primary sources if information for responders are reports from rescue personnel and reports from civilians. These reports may come from a 911 call or a responder calling in on a two-way radio to a dispatch center. This type of information flow as well as GPS transmission of responder locations are currently modeled in the DRIFTS.

Civilians are one of the greatest sources of information pursuant to an urban disaster. This is because there is often a large density of civilians in the disaster area, especially in an event such as an earthquake. These civilians are likely to be distributed in a similar fashion as the casualties of the disaster. Thus, civilians with cell phones or other means of communication can contact emergency response agencies to let them know about damage and casualties. However, civilians often respond with a greater degree of hysteria compared to emergency response personnel. Thus, the quality of civilian reports is likely to be more degraded then that of a police report, for example. Thus, as the civilians call in reports of damage and casualties, the attributes of the reports they give and confused and corrupted to a greater degree than that of response personnel reports.

These emergency personnel also give reports of observed damage and casualties. The police, for example, as 


\section{Robinson and Brown}

they drive through the city, report on what they can see out of their car. While the reports from police also get corrupted and confused, it is not to the degree of the civilian reports.

There are several methods of confusing the attributes of the reports. For categorical variables such as casualty severity or age category, a confusion matrix is used which gives a probability of a given reported value given an actual value. A Monte Carlo draw is then performed to give the appropriate confused value. Continuous variables such as location coordinates and the time the message is sent are altered according to some family of distributions. For example the location attributes are altered according to a normal distribution with the mean being the actual value. A normal random number is then drawn from a distribution with that mean and with the agent's associated variance. The variance is higher for civilians. This random draw is then reported. False reports are also sent alongside true reports. These false reports represent either things that people thought they observed but in fact did not, or severely confused reports which are simply wrong.

Lastly reports are also limited and corrupted. It is assumed that there is some random limit as to the number of reports that can be received by any one call center or other receiving facility. Thus, there is an upper limit as to the number of reports that can be sent. The reports that make it through to the call center are randomly selected from the possible reports that might be sent. Furthermore, some attributes in a report are also not sent at all. For instance, a location of a casualty might not be transmitted. This is representative of multiple people trying to talk on a radio at the same time or other types of communication failures that limits the amount of total information that is sent.

All of these corruptions and confusion in reports is meant to model the real reports that emergency facilities receive after a disaster. Many are incomplete reports and many are simply wrong. Thus, in DRIFTS, the information actually flowing through the agents not only has accurate and specific origins, but it is transferred to other agents as information might be transmitted in a true scenario.

\subsection{Simulation Contributions}

Currently DRIFTS is being used to aid in developing an efficient data fusion methodology. However, its potential contributions are many. DRIFTS currently has the capability for directing individual response agents throughout almost any geographic area for which one has GIS databases. Furthermore the agents have the built in capability of being dynamic in characteristic and location. For example, hospitals can fill up and a casualty's condition can get worse or better.

DRIFTS has also been developed with flexibility in mind. As new models of police movement or casualty dynamics evolve, they can be seamlessly and quickly integrated into the existing framework. Thus, as new models are validated, the simulation becomes closer to representing actual reality. This is accomplished through an agentbased framework with the flexibility of layering different agents within GIS.

\section{CONCLUSION}

Many researchers have identified the necessity of using computer simulation to model emergency response to disasters. Computer simulation has been suggested for use as training tools and to aid emergency mangers in preparation for future events. However, it can also be used to assess new technologies which might be used in a response environment. More specifically, computer simulation can especially aid in assessing the effects of information technologies. Information technologies are going to be increasingly important in emergency response operations as more and more data becomes available to emergency managers. This data must be converted into usable information and then disseminated properly so it can aid in response. This collection and subsequent dissemination is the information flow. This must be properly modeled and implemented in computer simulations to aid in the assessment of new information technologies.

Also, in the process of assessment, a combination of computer, human-in-the-loop, and live simulation can be used in a process that mimic response surface methods to find an optimal set of inputs which will generate optimal outputs. Within this framework, computer models can be roughly implemented and then later refined. In this process of refinement of models, an agent-based architecture gives a good level of flexibility and inexpensive refinement of the actual simulation.

Given the benefits of agent-based structure and the capabilities of GIS, a Disaster Response Information Flow and Technology Simulation has been developed which models the information flow in a disaster event. The disaster loss estimates are generated through FEMA's HAZUS. These are then transformed into a "ground truth". Agents are next simulated in this ground truth environment, scanning, reporting, and transporting casualties in a plausible manner. This information flow is necessary to model to assess information technology and DRIFTS has this capability.

\section{REFERENCES}

Balci, O. 1998. Verification, validation, and testing. Handbook of Simulation: Prinicples, Methodology, Advances, Applications, and Practice. New York, NY: John Wiley and Sons.

Box, George. 1979. Robustness in the strategy of scientific model building. In R. Launer \& G. Wilkinson (Eds.) Robustness in Statistics, cited by Temple, J. (1998) Robustness tests of the augmented Solow model, Journal of applied econometrics, 361-75. 


\section{Robinson and Brown}

Brady, Thomas F. 2003. Emergency management: Capability analysis of critical incident response. Proceedings of the 2003 Winter Simulation Conference. 1863-1867.

Gadomski, Adam M., Claudio Balducelli, Sandro Bologna and Giovanni DiConstanzo. 1998. Integrated parallel bottom-up and top-down approach to the development of agent-based intelligent DSSs for emergency mangement. Disaster and Emergency Management: International Challenges for the Next Decade. The Fifth Annual Conference of the International Emergency Management Society.

Guha-Sapir, Debarati and Regina Below. 2000. The quality and accuracy of disaster data: A comparative analysis of three global data sets. A study by the Provention Consortium.

Jain, Sanjay and Charles McLean. 2003. A framework for modeling and simulation for emergency response. In the Proceedings of the 2003 Winter Simulation Conference, eds. S. Chick, P. J. Sánchez, D. Ferrin, and D. J. Morrice. IEEE, Piscataway, New Jersey.

Jain, Sanjay, Charles McLean and Swee Leong. 2003. Modeling, simulation and visualization for emergency response. Draft report released at workshop on Modeling and Simulation for Emergency Response, March 4-6, 2003 at NIST, Gaithersburg, MD.

Jenvald, Johan, Magnus Morin and J. Peter Kincaid. 2001. A framework for web-based dissemination of models and lessons learned from emergency-response exercises and operations. International Journal of Emergency Management, 1 (1): 82-94.

Koehler, Gus. 1995. What disaster response management can learn from chaos theory. Ed. Gus A. Koehler. What Disaster Response Management Can Learn From Chaos Theory, May 1995, California. California Research Bureau.

Knepell, P.L. and D.C. Arangno. 1993. Simulation validation: a confidence assessment methodology. IEEE Computer Society Press.

Myers, Raymond H. and Douglas C. Montgomery. 2002. Response surface methodology. $2^{\text {nd }}$ Edition. New York: Wiley.

Sanchez, Susan M. and Thomas W. Lucas. 2002. Exploring the world of agent-based simulations: simple models, complex analyses. Proceedings of the 2002 Winter Simulation Conference. 116-126.

Sullivan, Thomas J. 1985. Modeling and simulation for emergency response. Lawrence Livermore National Laboratory, Report No. UCRL 92001 Preprint.

Wallace, W. A. and F. De Balogh. 1985. Decision support systems for disaster management. Public Administration Review, 45, Special Issue: Emergency Management: A Challenge for Public Administration, 134146. Washington D.C.: American Society for Public Administration, 1985.

\section{AUTHOR BIOGRAPHIES}

C. DONALD ROBINSON is currently enrolled in the Ph.D. program with the Department of Systems and Information Engineering at the University of Virginia. He has B.S. degrees in Physics, Mathematics, Computer Science, Economics, and Applied Mathematics from HampdenSydney College. He has held positions of Engineer, Sergeant and Lieutenant with the Hampden-Sydney Volunteer Fire Department. His research interests include data mining/fusion and simulation with applications to emergency response. donaldrobinson@virginia. edu.

DONALD E. BROWN Ph.D. is W.S. Calcott Professor and Chair of the Department of Systems and Information Engineering, University of Virginia. He is a Fellow of the IEEE and the Editor-in-Chief of the IEEE Transactions on Systems, Man, and Cybernetics, Part A: Systems and $\mathrm{Hu}-$ mans. He is the recipient of the IEEE Norbert Wiener Award, the IEEE Intelligence and Security Informatics research achievement award, and the IEEE Millennium medal. Dr. Brown received a Ph.D. from the University of Michigan in Industrial and Operations Engineering. He does research in data fusion, statistical learning, and behavior discovery and simulation with applications to security and safety. Brownevirginia.edu. 